\title{
Effect of Atomic Hydrogen Exposure on Electron Beam Polarization from Strained GaAs photocathodes
}

\author{
M. Baylac, P. Adderley, J. Clark, T. Day, J. Grames, J. Hanskneckt, \\ M. Poelker, P. Rutt, C. Sinclair, M. Stutzman \\ Thomas Jefferson National Accelerator Facility \\ 12000 Jefferson Avenue, Newport News, VA 23606
}

\begin{abstract}
Strained-layer GaAs photocathodes are used at Jefferson Lab to obtain highly polarized electrons. Exposure to atomic hydrogen (or deuterium) is used to clean the wafer surface before the activation with cesium and nitrogen trifluoride to consistently produce high quantum yield photocathodes. The hydrogen-cleaning method is easy, reliable and inexpensive. However, recent tests indicate that exposure to atomic hydrogen may affect the polarization of the electron beam. This paper presents preliminary results of a series of tests conducted to study the effect of atomic $\mathrm{H}$ exposure on the polarized electron beam from a strained-layer GaAs sample. The experimental setup is described and the first measurements of the beam polarization as a function of exposure dose to atomic hydrogen are presented.
\end{abstract}

\section{INTRODUCTION}

Jefferson Lab is a nuclear physics research facility where highly polarized electrons can be delivered to three experimental endstations. The beam is produced by photoemission from strained layer GaAs photocathodes, optically pumped by circularly polarized laser light. The photocathode is formed, or activated, with Cs and NF3 to build a NEA surface. Preparation of an atomically clean surface is an essential step in the fabrication of an NEA surface.

Wet chemical etching techniques were initially used at Jlab but results often varied, something we attribute to variations in chemical quality and purity. The method of hydrogen cleaning, which is widely used in the semiconductor industry [1], was implemented at JLab and provided a drastic improvement in our ability to create photocathodes with consistently high QE. The method is particularly well suited for cleaning thin strained layer photocathode samples because, in principle, only contaminants are removed from the surface. Other laboratories recognize the benefits of hydrogen cleaning; we have helped implement this cleaning method at MAMI, Nagoya, Bates and SLAC.

Despite obtaining high $\mathrm{QE}$ from strained layer photocathodes, our initial experience with high polarization photocathodes was frustrating. Polarization from some samples was often very low $(\sim 50$ to $60 \%)$ and polarization could vary from a single sample as the laser beam was moved across the active area of the photocathode (few mm's). 
Over time, at least some of this behavior was attributed to excessive cleaning with atomic hydrogen. This paper presents results from a dedicated experiment to quantify the effects of hydrogen exposure on beam polarization from a single photocathode sample.

\section{EXPERIMENTAL SETUP}

The photocathode sample used for this test was obtained from Bandwidth Semiconductor (formerly Spire Corporation) and was grown to SLAC specifications [2]. The sample was cleaved to proper dimensions $(15.5 \mathrm{~mm} \times 15.5 \mathrm{~mm})$ and indium soldered to a standard JLab stalk. The sample was not treated with wet chemicals at any time throughout the experiment (no acid/base etching, degreasing or anodization).

Beam polarization and $\mathrm{QE}$ measurements were performed on a test stand that includes a $-100 \mathrm{kV}$ photogun and Mott polarimeter. Hydrogen cleaning was performed on a separate vacuum chamber that is used for cleaning all of the photocathode samples used at the CEBAF photoinjector. When not in vacuum, great care was taken to ensure the sample was exposed only to clean, inert nitrogen. Venting of the vacuum chambers was conducted using nitrogen filled glove bags and the sample was transferred between chambers within a nitrogen filled transport vessel.

The hydrogen cleaning vacuum chamber consists of a 6 way cross with 4.5 " flanges [3]. There are two vacuum pumps; a $20 \mathrm{~L} / \mathrm{s}$ Perkin Elmer ion pump and a Balzers 50 $\mathrm{L} / \mathrm{s}$ turbo pump which serves as the dominant pump during hydrogen cleaning. Molecular hydrogen (or deuterium) flows through a leak valve into a Pyrex glass dissociator. The molecular hydrogen is dissociated with an RF inductive discharge created by coil, which is part of a tuned LC circuit. The LC circuit resonates around $100 \mathrm{MHz}$ and $20 \mathrm{~W}$ of RF power is absorbed by the hydrogen when the dissociatorregion pressure is $15 \mathrm{mTorr}$. Atomic $\mathrm{H}$ exits the dissociator through a $1 \mathrm{~mm}$ diameter hole to reach the photocathode approximately $15 \mathrm{~cm}$ away, at a temperature $\sim 300^{\circ} \mathrm{C}$.

On the beam teststand, a high power DC Nd:YVO4 laser pumps a Titanium:Sapphire crystal inside a four-fold optical cavity. The Ti:Sap laser light is wavelength tunable between 740 and $860 \mathrm{~nm}$. It is circularly polarized by a Pockels cell and the helicity of the beam is reversed pseudo-randomly at $10 \mathrm{~Hz}$. Laser light enters the gun chamber through a vacuum window to reach the photocathode. The vertical electron gun consists of a sample loading section, a preparation chamber and a high voltage section [5]. An isolated load lock chamber enables loading of a stalk while keeping the gun under vacuum. This small chamber can be quickly pumped and baked. The preparation chamber, or main gun chamber, accommodates the different ports necessary to perform the NEA activation of the semiconductor surface. An alumina ceramic is used to hold off the $100 \mathrm{kV}$ acceleration voltage. The stalk is placed in contact with the cathode seating at high voltage. Electron beam exits the gun through the center of a donut-shaped anode. It travels through a simple beam line consisting of a bend magnet, correctors and lenses, beam viewers and an electrostatic bend used as a spin rotator. At the end of the beam line, electrons impinge on a gold foil in the Mott chamber. The Mott polarimeter contains two identical silicon detectors in the horizontal plane tracking electrons backscattered $\left(\theta= \pm 120^{\circ}\right)[6]$. Measuring the 
experimental counting rate asymmetry between the 2 helicity states and/or between the 2 detectors yields the electron beam polarization, when one knows the analyzing power of the polarimeter ( $\sim 33 \%$ for Mott scattering off a 300 A gold foil at $100 \mathrm{keV})$.

\section{EXPERIMENT}

The experiment consisted of first characterizing the sample prior to exposure to atomic hydrogen. Following this, the sample was repeatedly exposed to atomic hydrogen in $\sim 15$ minute time intervals for a total cumulative dose of 100 minutes. The experiment was time consuming, requiring successful implementation of many steps including; a) sample loading into the $-100 \mathrm{kV}$ gun, b) vacuum chamber bakeout, c) sample heating, d) sample activation, e) beam measurement of polarization and $\mathrm{QE}$ versus wavelength, f) vacuum chamber venting and sample transport to the hydrogen cleaning chamber, g) hydrogen cleaning and then finally reinstallation of the sample into the $-100 \mathrm{kV}$ gun where the process repeats. Every effort was made to ensure that the steps were carried out under identical conditions following successive hydrogen exposures. Some specific details on individual steps of the test are given below.

After the sample is installed in the load lock chamber of the test gun, this chamber is evacuated and baked at a temperature around $250^{\circ} \mathrm{C}$ for 12 hours. After cool down to room temperature (RT), the load lock chamber is open to the main chamber of the electron gun and the stalk holding the photocathode inserted into the gun. The sample is then heated again to $\sim 570^{\circ} \mathrm{C}$ for 2 hours. We activate the wafer after it cooled down to RT in the gun chamber. Approximately one monolayer of cesium is applied to the GaAs surface and oxidized by nitrogen trifluoride (NF3) while photocurrent yield is measured during illumination with white light.

Once the characterization is complete (QE, polarization), the sample is retracted from the gun chamber into the load lock chamber. The load lock chamber is vented to atmospheric pressure while the gun and the beamline remain under vacuum at all times. The stalk is transported and loaded into the chamber equipped with the hydrogen dissociator; the chamber is pumped down. Once the pressure reaches $\sim 10-8$ Torr, the sample is heated to $500^{\circ} \mathrm{C}$ for a few minutes and quickly cooled to $300^{\circ} \mathrm{C}$. Atomic deuterium is released into the chamber at a pressure of $15 \mathrm{mTorr}$ and the dissociator parameters are adjusted quickly while a paddle on top of the glassware protects the sample surface from the gas flow. Once the RF power absorbed by deuterium is maximized $(\sim 40 \mathrm{~W})$, the paddle is removed and the ion counter is engaged. The RF power is switched off and the sample is cooled down as the ion

count reaches the desired value corresponding to a 15 minute cleaning time. The sample is transferred back into the gun for the next series of measurements.

\section{RESULTS}

Polarization and QE results for the unexposed sample are shown in Fig. 1. It is interesting to note that $\mathrm{QE}$ is quite good $(0.35 \%$ at $840 \mathrm{~nm}$ of incident laser light), even without hydrogen dose, indicating that our methods for handling samples may have improved at Jlab (photocathode cleaning, in one form or another, was essential to 
obtain high QE years ago). The QE of the photocathode surface is scanned and is measured to be uniform within $\sim 10 \%$. Polarization measurements were made at 5 cathode locations. Conditions were kept constant for all measurements (foil thickness, beam steering, counting rates) to minimize systematic discrepancies between different runs. The systematic uncertainty of the absolute polarization with this Mott polarimeter is estimated $\sim 10 \%$, the relative comparison of one measurement to another is smaller. A maximum polarization $\sim 80 \%$ is measured at $840 \mathrm{~nm}$.
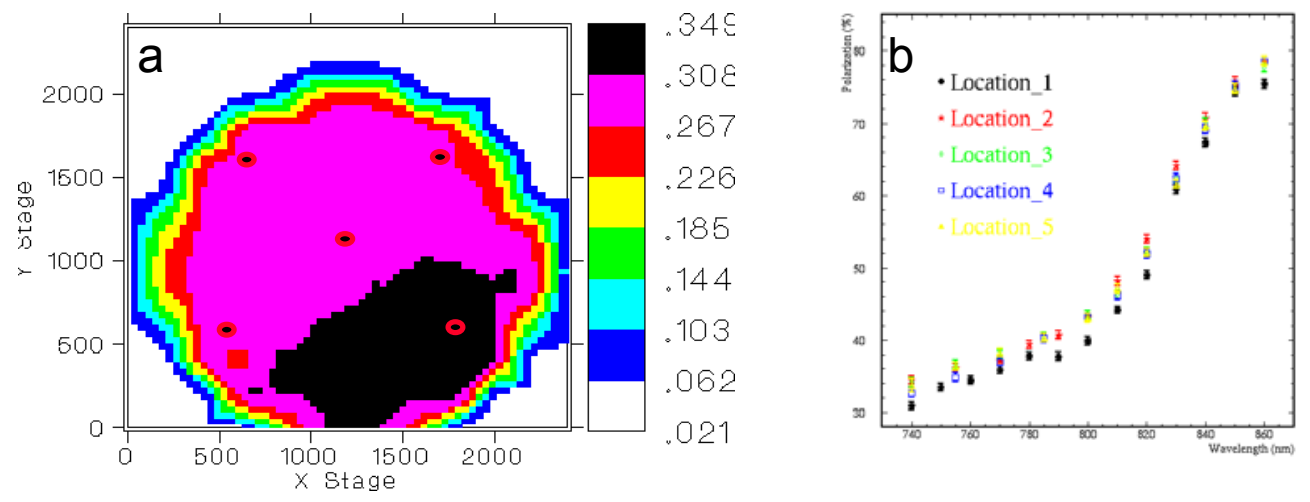

FIGURE 1. (a) Quantum efficiency profile of the photocathode at $840 \mathrm{~nm}$ and 5 locations selected for polarization measurements; (b) Beam polarization vs. wavelength for the 5 locations (statistical errors only).

Polarization as a function of hydrogen dose is shown in Fig. 2 for locations 1 and 3. We observe a strong depolarization as the $\mathrm{H}$ dose is increased (60 minutes and more). This effect varies significantly with wavelength: the relative polarization variation $\Delta \mathrm{P}(\mathrm{t})=[\mathrm{P}(\mathrm{t})-\mathrm{P}(0)] / \mathrm{P}(0)$ where $\mathrm{t}$ is the cleaning time, is maximum at high wavelengths whereas $\Delta \mathrm{P}$ exhibits very little dependence below the band-gap $(<800 \mathrm{~nm})$. However, a minimal $\mathrm{H}$ dose may seem to increase slightly the polarization, $0<\Delta \mathrm{P}(\mathrm{t})<+10 \%$.
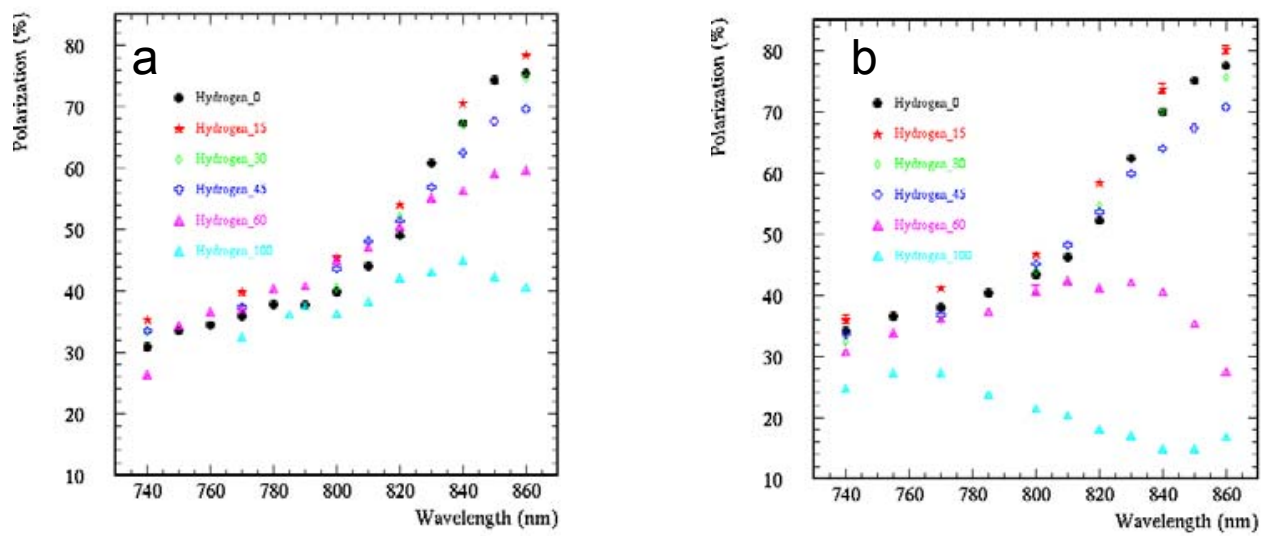

FIGURE 2. Beam polarization vs. wavelength for 6 total $\mathrm{H}$ exposure $(0,15,30,45,60$ and 100 minutes) for locations 1 (a) and 3 (b). Errors are statistical only.

Moreover, the depolarization varies strongly across the surface: $-80 \%<\Delta \mathrm{P}(\mathrm{t})<-40 \%$ for the 5 measured locations whereas the initial polarization profile was uniform. 
The quantum yield is measured to be decreasing as the $\mathrm{H}$ dose increases: from $0.035 \%$ at $840 \mathrm{~nm}$ with a bare surface down to $0.002 \%$ after the photocathode has been exposed to the maximum dose. This trend was confirmed by a similar test performed in a different chamber equipped with a dissociator, which remained under vacuum throughout all measurements.

The original sample used for this test was removed from the gun and sent for Atomic Force Microscope imaging. The surface analysis indicates some differences when compared to a bare uncleaned strained GaAs sample but did not provide any conclusive results regarding structure changes of the semiconductor surface.

\section{CONCLUSIONS \& OUTLOOKS}

This experiment carried out the first characterization of the polarization of an electron beam photoemitted from a strained layer GaAs semiconductor as a function of atomic hydrogen exposure of the surface. The preliminary results show that the hydrogen cleaning process of our photocathodes needs to be revisited. Indeed, exposure to a heavy dose of hydrogen can depolarize the sample in a dramatic manner. A strong dependence on the wavelength of the incident light is also observed, but not yet understood, as the depolarization increases significantly with wavelength. The past benefit of this cleaning procedure is called into question, as wafers now provide high QE directly from manufacturers. This experiment brings some new light on intriguing past measurements (low polarization, non-uniformity) as poor performances can now be attributed to overexposure to hydrogen. However, one may also notice indications of a slight increase in polarization with a small $\mathrm{H}$ dose, which can create some new interest in the $\mathrm{H}$ exposure.

Since this test was done, a hydrogen dissociator has been installed on the preparation chamber of the test gun to continue the experiment. We will repeat this test with a strained layer GaAs sample to confirm the observed behavior. A similar series of measurements is in progress using a bulk GaAs wafer. This aims to pinpoint the origin of the depolarization mechanism, as it will help differentiate between effects associated with the surface or with the strain of the semiconductor. Study is also underway regarding the characteristics of the $\mathrm{H}$ source, as the energy and angle of incidence onto the wafer surface of the atomic hydrogen may play a significant role. Optimizing the $\mathrm{H}$ cleaning source parameters may help reaching atomically flat surfaces. Whereas the use of atomic $\mathrm{H}$ for cleaning semiconductors seems now irrelevant, it may become a tool to increase the beam polarization.

\section{REFERENCES}

1. E.J. Petit et al, J.V.S.T. A 10, 2172 (1992); E.J. Petit \& F. Houzay, J.V.S.T. A 12, 547 (1994)

2. C.K. Sinclair et al, proc. 1997 PAC

3. M. Poelker et al, proc. 1997 JLab Contamination workshop: measurement and control in vacuum sys.

4. W.W. Mc Alpine, R.O. Schildknecht, proceedings of the IRE, 2099 (1959)

5. B. Dunham, Ph. D thesis, University of Urbana-Champaign, Illinois (1993) 